\title{
Modelling and Simulating Exit Selection during Assisted Hospital Evacuation Process using Fuzzy Logic and Unity3D
}

\author{
Intiaz Mohammad Abir, Ali Ahmed Ali Moustafa Allam, Azhar Mohd Ibrahim* \\ Department of Mechatronics Engineering \\ International Islamic University Malaysia \\ Kuala Lumpur, Malaysia
}

\begin{abstract}
Evacuation procedures are an integral aspect of the emergency response strategy of a hospital. Evacuation simulation models help to properly evaluate and improve evacuation strategies. However, the issue of exit selection during evacuation is often overlooked and oversimplified in the evacuation simulation models. Moreover, most of the available evacuation simulation models lack integration of movement devices and assisted evacuation features. However, finding a solution of these limitations is a necessity to properly evaluate evacuation strategies. To tackle this problem, we propose an effective approach to model exit selection using a fuzzy logic controller (FLC) and simulate assisted hospital evacuation using Unity3D game engine. Our research demonstrates that selecting exits based on distance only is not sufficient for real life situation because it ignores the unpredictability of human behavior. On the contrary, the use of the proposed FLC for exit selection makes the simulation more realistic by addressing the uncertainty and randomness in an evacuee's decision-making process. This research can play a vital role in future developments of evacuation simulation models.
\end{abstract}

Keywords-Evacuation simulation; exit selection; fuzzy logic; unity $3 D$

\section{INTRODUCTION}

Hospitals are generally called a safe building and an emergency care centre for individuals. Planning for disasters is essential to reduce the number of disaster-related deaths and injuries. However, Planning a well-timed evacuation of complex buildings such as hospitals is difficult. Hospitals have many patients with fractures or injuries. Majority of them face evacuation difficulties as they must use wheelchairs or crutches and can't move normally.

It can be asserted that the behavioural reaction of the evacuees throughout the movement plays a vital role in the evacuation process. The modelling of the movement of the evacuees which is correlated with behavioural reaction has been given importance while designing evacuation models. For safe evacuation, the importance of designing evacuation models to predict the evacuation process is paramount [1] Some of these models are; SGEM [2], [3], [4], SIMULEX [5], [6] EXODUS[7] , EGRESS [8], [9]. Behavioural reaction plays a major impact in the evacuation process and choosing an exit is a comparatively complex factor while evacuating. The term exit not only refers to the final exit which leads outside the structure but also internal exits which lead the evacuees from one confined area to another. As there are several exit options available in a multi-exit structure, the evacuees face a major dilemma while choosing the right exit. In a situation where evacuation is necessary, everything occurring in the environment works as stimuli and shapes the reaction of the evacuees (e.g., the activity of other evacuees [1]).

Today's evacuation simulation software allows designers to easily evaluate evacuation performance for various conditions and designs of the building's internal structure. Over the years, a vast number of models have been developed for general building evacuation simulation. However, it could be impossible or inappropriate to use most of these models for simulating hospital evacuation as it is necessary for hospital environments to include movement devices (e.g., wheelchair and crutches) and assisted evacuation features (i.e., the ability to assist wheelchair users to evacuate). Moreover, proper modelling of exit selection is needed to make the simulation process smoother. Hence, we propose an effective approach to select a proper exit for each evacuee agent (doctors, nurses, patients, or visitors) using a fuzzy logic controller (FLC) and develop an assisted hospital evacuation simulation model using Unity3D game engine.This model can be used by the hospital authorities to calculate the total evacuation time of the evacuees. In this way, the efficiency of evacuation measures will also be quantified which will lead to a safer evacuation planning and designing.

The rest of the paper proceeds as follows: Section II explores previous research related to our work. Section III explains the methodology and implementation of this research. Section IV analyses and discusses the results. Finally, Section $\mathrm{V}$ provides the conclusion of the research.

\section{RELATED WORK}

There are many evacuation models that can simulate the evacuation process of a general building. However, most of these models lack proper integration of movement devices and assisted evacuation features as these models were developed to simulate ambulant evacuees. Some of the current evacuation models which are not explicitly designed to simulate assisted evacuation but are sufficiently flexible to accomplish this purpose indirectly. Disabled people were included in certain models by reducing their speeds. For example, FDS+Evac [10] model simulates the evacuation of elderly agents at a lower speed than normal agents. The assisted evacuation of hospitals and wheelchairs was only investigated by a small range of studies. For example, Hunt et al. [11], Alonso et al. [12], 
Ursetta et al. [13], and Rahouti et al. [14] simulated assisted evacuation procedures in a hospital environment. However, these models lack proper modelling of exit selection.

It is important to take exit selection on a serious note as the behavior of an individual has considerable impact in the evacuation process and also has the potential to cause another emergency [15]. Complications arise when there is an imbalance in the evacuee crowd. If the structure has uneven design of exits it will result in an imbalance in the number of evacuees in the exits [16]. Researchers demonstrated that some components have direct impact on the evacuees' decision making such as, exit distance, the decision of following other individuals, the exit being in the range of vision, availability of light in the surrounding, crowd distribution, human psychology, width of the doors, the capacity of a particular exit , obstacle position, the queue length in the exits, familiarity with the structure, density of the crowd in particular exits, exit familiarity, angle made by the exit in respect to current movement direction, movement direction of other evacuees and social influence [1], [17], [18], [19], [20], [21], [22], [23], [24] . A study by Lovreglio et al. [25] demonstrated that in certain circumstances evacuees crowd up to particular exits avoiding other available exits. Wang et al. [26] analyzed the behavior of evacuees during a panic situation by using a combination of automata and multi-agent based model and demonstrated that the selection of exit behavior varies based on the crowd around that exit. These findings were also validated by $\mathrm{Xu}$ et al. [27].

Many models have been developed by researchers in past decades in order to regulate exit selection during evacuation [28], [29], [30], [31], [32]. Dynamic background field [33], [34], bayesian game theory [35], simulated annealing (SA) and depth-first search (DFS) [36] are some of the proposed methods to solve this issue. The selection of exit is made by considering the reciprocity of the group of evacuees in a game theory based model proposed by Lo et al. [1] which can be considered as one of the most notable works done in this research field.

In recent years, researchers have adopted methods like fuzzy logic, least effort algorithm, game theory, random utility theory, modified multinomial logit model, reinforcement learning to model exit selection. For example, Yang et al. utilized fuzzy logic to model exit selection using two input parameters: Normalized distance and normalized density [37]. Liu et al. followed a similar approach like Yang et al. However, they combined the output of the fuzzy logic system with exit width and herding behavior to determine the target exit [38]. Wang et al. utilized game theory to model exit selection. $\mathrm{He}$ considered factors like distance, visual range and choice firmness [39]. Zhang et al. proposed a multi-exit selection model considering three factors: distance, density and exit width [40]. Fu et al. proposed Two multi-exit selection models based on the social force model to analyze the dynamic change in exit selection by considering the effect of the exit distance, exit width, crowd number and crowd distribution [41]. Xu et al. proposed deep reinforcement learning based exit selection model named MultiExit-DRL [42]. Cao et al. implemented exit selection model based on random utility theory [15]. Edrisi et al. proposed three different exit choice models which are: the shortest path exit choice, the multinomial logit model, and the modified multinomial logit model with revising decisions. Their research demonstrated that the modified multinomial logit model with revising decisions outperforms the other two models [43]. Ma et al. modified the social force model and proposed an integrated exit selection model where evacuees can observe nearby evacuees and choose the appropriate exit by calculating the shortest estimated evacuation time [44]. Fu et al. combined least effort algorithm with a cellular automaton model to model exit selection while considering the distance to exits and crowd density around exits [45].

The movement of the evacuees can be distributed in three levels and among them, exit choice belongs to the highest strategic level. Mostly it is presumed that the exit selection is done entirely based on shortest distance optimization [46], [47], [48]. However, considering only distance is not enough to properly model the exit selection problem as in real time situations evacuees consider other relevant factors as well. Moreover, from the above review we can conclude that most of the existing exit selection models considered environmental factors (e.g., exit width, crowd density) while ignoring psychological factors (e.g., exit familiarity). Previous works/experiments suggest that factors like familiarity [18], [24] and visibility [22], [23], [24] can play a vital role in exit selection as human behavior is unpredictable and does not depend on one single factor. Thus, a simpler method is necessary for helping the evacuee agents in quick decision making. This paper proposes a new approach which considers psychological factor (exit familiarity) and environmental factors (exit distance and exit visibility) of exit selection by using a Fuzzy Logic Controller (FLC). Hence, it will be able to address the unpredictability of human behavior to some extent. These three factors are taken from the available literature that were discussed previously. This method will be able to provide a more realistic evacuation simulation compared to other methods where exit selection is done solely based on distance or on random.

\section{Methods}

Evacuation simulation models are important for investigating various evacuation strategies suitable for different scenarios. Reviews of general evacuation simulation models indicate that these models were mainly developed for normal building environments where evacuees can usually move unaided. Only a few studies are available on assisted evacuation.

In this paper, we propose an assisted evacuation simulation model to simulate the evacuation process of a hospital where assistants transfer non-ambulant patients from a risky place to a safe place using hospital devices such as wheelchairs and other ambulant evacuees (e.g., Doctors, Nurses, patients, and visitors) evacuate on their own. The proposed model utilizes a FLC to regulate the exit selection behavior of the ambulant evacuees (e.g., Doctors, Nurses, patients, and visitors). This section describes the details of the proposed system design.

\section{A. Software}

The simulation was carried out using Unity3D which is a game development engine developed by Unity Technologies. This software is capable of building 3D, 2D, Virtual Reality games, simulations, and other interactions. Fuzzy logic 
modelling was done using MATLAB and later implemented in Unity3D. Finally, 3DS Max, which is a professional 3D graphics computer tool designed to create 3D animations, models, and games was used to design the agents and the movement devices

\section{B. Types of Agents}

Occupants with different levels of dependence will be expected at health facilities. To better simulate the process of evacuation in this form of setting, it is important to introduce two types of agents: ambulant agents such as patients, employees or visitors who can move without help, and non-ambulant agents such as dependent and highly dependent patients. For the simulation, six types of agents were considered. These are: doctors, nurses, visitors, patients with crutches, dependent patients with wheelchairs, independent patients with wheelchairs. Fig. 1 illustrates the different agents.

\section{Movement Devices}

Explicit representation of movement devices is a necessary requirement to simulate the evacuation of health facilities. Two types of movement devices were considered for this simulation. These are wheelchairs and crutches. The wheelchairs can be operated in two ways. In the first case the patient is disabled but independent. Which means the patient can operate the wheelchair on its own. In the second case the patient is both disabled and dependent, so assistance is required from ambulant agents (e.g., staff, nurse) to move the wheelchair. On the other hand, crutch users can move on their own without requiring any assistance. The usage of movement devices is illustrated in Fig. 1.

\section{Layout Design}

In this paper, the simulated hospital area is a $\mathrm{W} \times \mathrm{H}$ hall with $40 \mathrm{~m} \times 60 \mathrm{~m}$ dimension. The considered floor has twentyone rooms for patients including beds, one room for nurses, cafe, one room for dentist, one room for X-ray, one room for

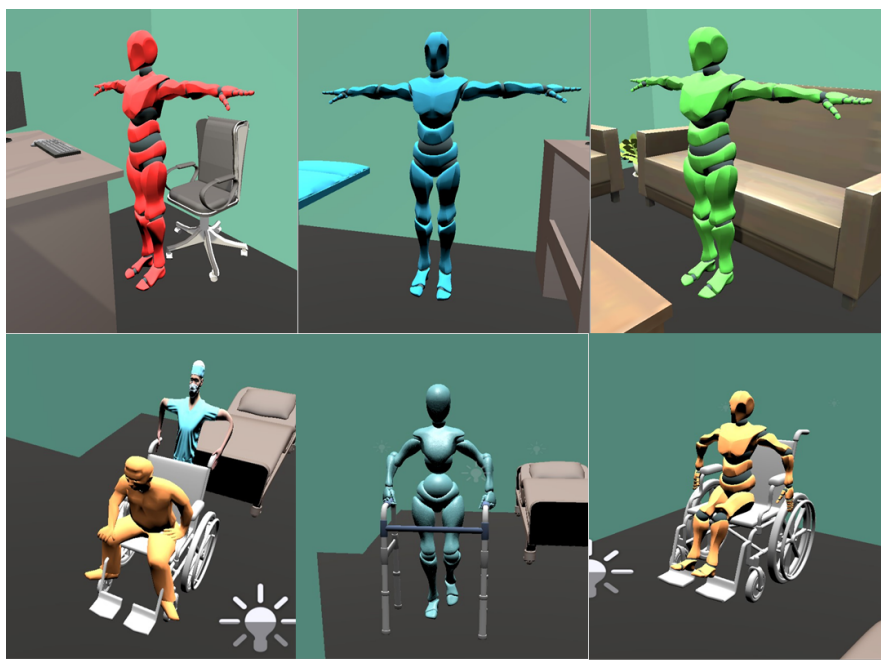

Fig. 1. Agents and Movement Devices used for Simulation. Doctors(red), Nurses(blue), Visitors(green), Patients with Crutches, Independent Patients with Wheelchair, Dependent Patients with Wheelchair. general practitioner and reception. Fig. 2 shows the layout of the simulation area.

\section{E. Agent's Navigation System}

For this research, we used Unity's built-in navigation system named NavMesh AI [49] which is divided into two main components. The first one is NavMesh (Navigation Mesh) area, which is used to define navigable areas in the environment. This component specifies areas where agents can walk, as well as the position of obstacles that the agent needs to avoid. This system is used for pathfinding and AI-controlled navigation. The second one is the NavMesh Agent component which is used to define an object as agent and also to set the agents' characteristics and features. This NavMesh AI helps agents to avoid each other, move around the environment towards a goal/target (e.g., exit). The agents can only move in the walkable surfaces while avoiding the obstacles and other agents. In Fig. 2 the blue area is the NavMesh area which is the agents' walkable surface. No agent can navigate in the remaining area. The walls and the furniture are obstacles for agents and the agents must avoid them while navigating.

\section{F. Modelling Exit Selection}

Most of the classical mapping or classification techniques, regardless of the number of classes or sets can be normalized down to two sets or classes namely 0 (false) and 1 (true). When an element belongs to a set, binary mapping evaluates it as 1 or true. If an element does not belong to a set, it is evaluated as 0 or false. Because of their objective nature traditional mapping methods only apply to exact correspondence. But in real life there are many relationships which are not so black and white. So, these relationships can't be described using the traditional binary mapping techniques. Fuzzy logic however can be utilized to solve this issue to some extent. It is a mathematical modelling method based on fuzzy sets and the related membership functions. In fuzzy logic "degrees of truth" is taken into consideration rather than exact true or false (1 and 0). Unlike crisp sets, a fuzzy set allows partial belonging to a set, that is defined by a degree of membership, denoted by $\mu$, that can take any value from 0 (element does not belong at all in the set) to 1 (element belongs fully to the set).

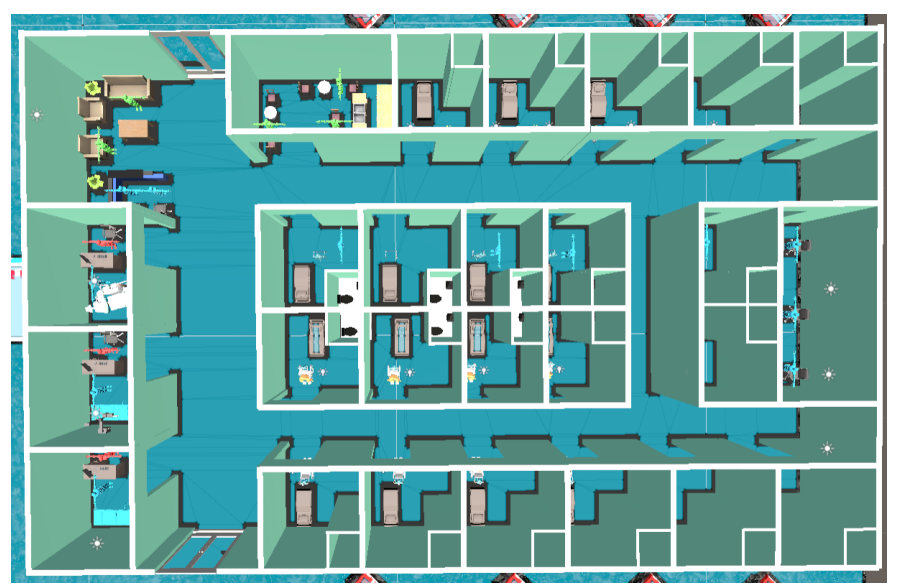

Fig. 2. Layout of the Simulation Environment. 
Everything in between 0 and 1 denotes the extent to which the element belongs to one set or the other. A fuzzy system is a repository of the fuzzy expert knowledge that can interpret data in subjective terms instead of precise Boolean logic. The expert knowledge is a collection of fuzzy membership functions and a set of fuzzy rules, known as the rule-base, having the form: IF (conditions are fulfilled) THEN (consequences are inferred)

Fuzzy logic is proposed in this paper to deal with human behavior uncertainty while selecting the proper exit during evacuation. As these parameters are hard to quantify using crisp calculation. The integration of fuzzy logic into computer models produced positive results where perception, feelings and judgment play a significant role. Therefore, human thinking and environmental adjustments are integrated in the proposed model using fuzzy laws to model the evacuee agent's exit selection behavior during the evacuation.

There are four major parts of a typical Fuzzy Logic Controller, namely fuzzifier, rule-base, inference engine and defuzzifier.

The fuzzifier performs fuzzification which is the process of converting crisp input values into fuzzy data points. In this process fuzzifier obtains the membership degree of the fuzzy set from the specific input according to the membership function. For this research, we have utilized triangular membership functions for all the linguistic variables.

After attaining the degree of membership for each input value from the fuzzifier, a rule-base is needed which contains the if - then rules/conditions that are required to form the inference process of the output variable. Generally, the rules are expressed by the fuzzy natural language. The number of fuzzy rules needed can be determined by multiplying the number of linguistic variables of all the inputs, for example, our proposed FLC has 3 input variables: Distance (four linguistic variables), visibility (three linguistic variables) and familiarity (three linguistic variables) so a total of $(4 \times 3 \times 3)=36$ if -then rules are required for the rule base. The rule-base of our proposed FLC is demonstrated in Table I.

The Inference Engine provides the decision-making logic of the controller. By applying the fuzzy rules of inference, it evaluates the fuzzy input values and the provided rules to deduce the fuzzy output values. For this research, we have adopted a typical Mamdani fuzzy inference engine [50], [51].

Finally, the defuzzifier converts the fuzzy output into quantifiable and objective crisp output. In this paper the output of the defuzzifier is the probability of choosing a certain exit. The centroid defuzzification method is used for the defuzzification process of our proposed FLC.

Our proposed FLC has three input variables and one output variable. These are described below:

\section{1) Inputs:}

Visibility: Visibility represents the visibility of the exits which may vary due to different smoke conditions or crowd density. The visibility of each exit is assigned randomly. The variable has three linguistic variables: Low Visibility (LV), Medium Visibility (MV), High Visibility (HV). The range of visibility is from $0-10$.
Distance: This variable provides the distance between an agent and an exit. The variable has Four linguistic variables: Very Near $(\mathrm{VN})$, Near $(\mathrm{N})$, Far $(\mathrm{F})$, Very Far (VF). The range of distance is from $0-45$ meter.

Familiarity: Familiarity represents how familiar an agent is with a specific exit. Same as visibility, familiarity is also selected randomly. The range is from $0-10$. The variable has three linguistic variables: Not Familiar (NF), Familiar (F), Very Familiar (VF). Figure 3 visualizes the input variables

\section{2) Output:}

Probability of Exit Selection: This variable determines the selection probability of an exit. The variable has 8 membership functions: Zero Probability (Z), Very Low (VL), Medium Low (ML), Low (L), Medium High (MH), High (H), Very High $(\mathrm{VH})$, One (O). Fig. 4 visualizes the output variable.

\section{Results And Discussion}

This section describes the result of the simulation and discusses the findings of this research. At the beginning, the agents were placed randomly in the simulation area. When the simulation begins, the unity NavMesh system allows the agents to move into the target point (e.g., exit). The FLC determines the exit for each agent. The simulation ends when all the agents evacuate from the floor. Fig. 5 shows the initial and the final position of the agents. The simulation was carried out by considering two cases.

In the first case the proposed FLC was not included. In the second case the process was again repeated with the inclusion of the FLC. A total of 44 agents were simulated in the environment ( 3 doctors, 15 nurses, 5 patients with crutches, 11 patients with nurses, 5 patients with wheelchairs and 5 visitors).

For simplicity and ease of visualization only the results of 10 randomly picked agents were included in the tables (1 doctor, 2 nurses, 2 patients with crutches, 2 patients with nurses, 2 patients with wheelchair, 1 visitor). The simulation was conducted 10 times for each case. The details of the simulations are provided below.

\section{A. Results}

1) Case 1 (Without using FLC): The simulation is conducted without the FLC for the first experiment. The speed of each agent was set to $1.4 \mathrm{~m} / \mathrm{s}$. The agent will choose the nearest exit as we have only considered distance in this case. Table II provides the simulation results of 10 randomly picked agents for the first experiment. The table shows the evacuation time (in seconds) of each agent for each simulation run and the standard deviation of the evacuation time

2) Case 2 (Using FLC): For the second experiment, the appropriate exit selection was done by the proposed FLC instead of selecting the nearest exit. The FLC takes the distance, visibility, and familiarity of each of the exits from each agent to the exits and calculates the probability of selection for each exit. The exit with the highest probability of selection is chosen as the target for each agent. Similar to case 1 The speed of each agent was set to $1.4 \mathrm{~m} / \mathrm{s}$. Table III provides the simulation results for the second experiment. 


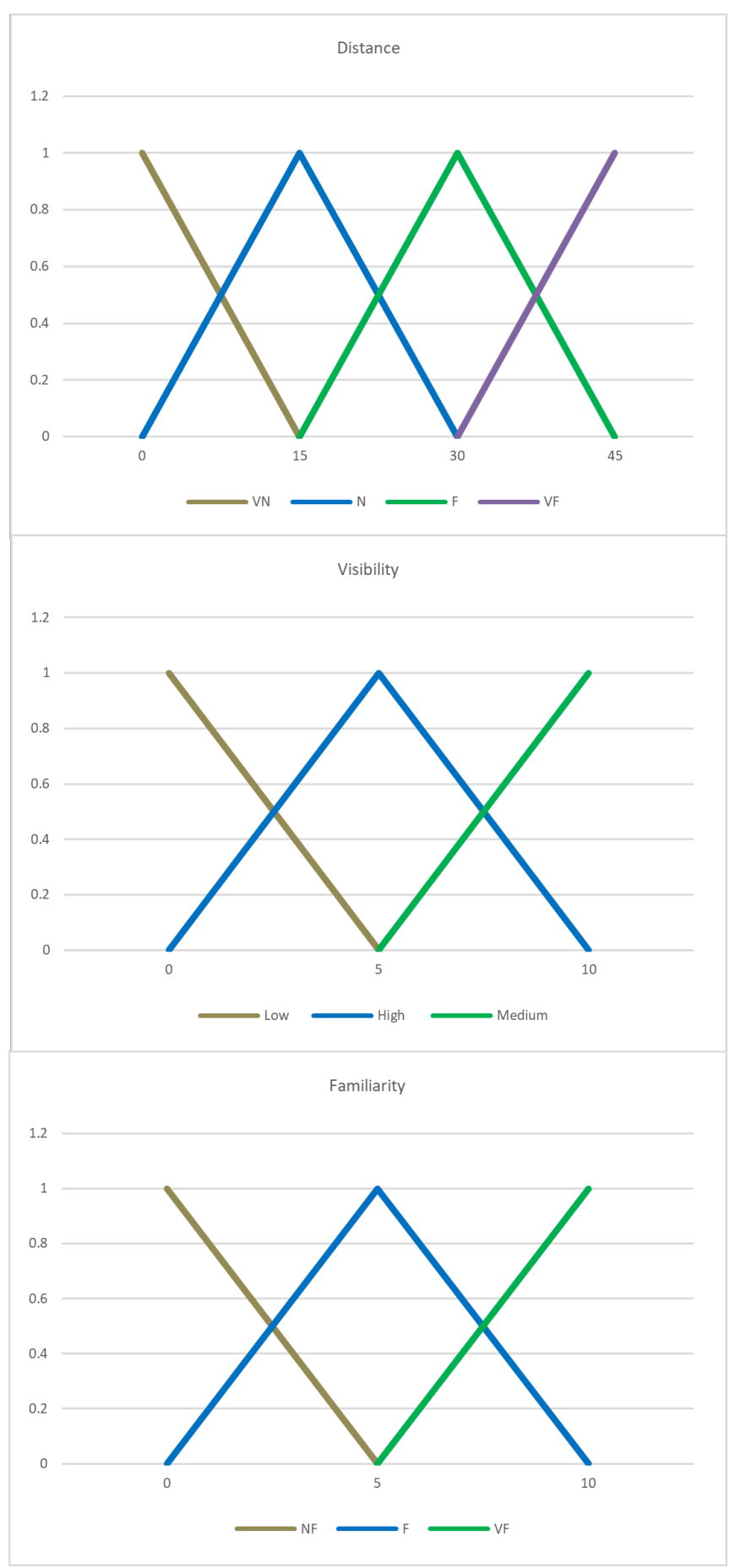

Fig. 3. Membership Function for Input Variables (Visibility, Distance, and Familiarity).

\section{B. Discussion}

When the exit is selected based on only distance the simulation fails to reflect human uncertainty and randomness. From Table II, it can be seen that the evacuation time of the agents remained almost the same for each test run and the standard deviation of the evacuation time is close to 0 . On the contrary, when the FLC is utilized, the agents selected the exit based on distance, familiarity and visibility which

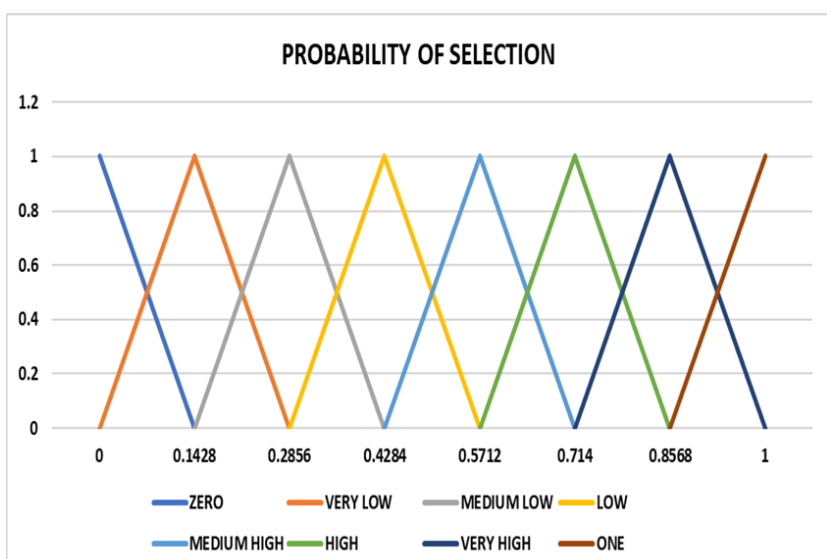

Fig. 4. Membership Function for Output Variable (Probability of Exit Selection).

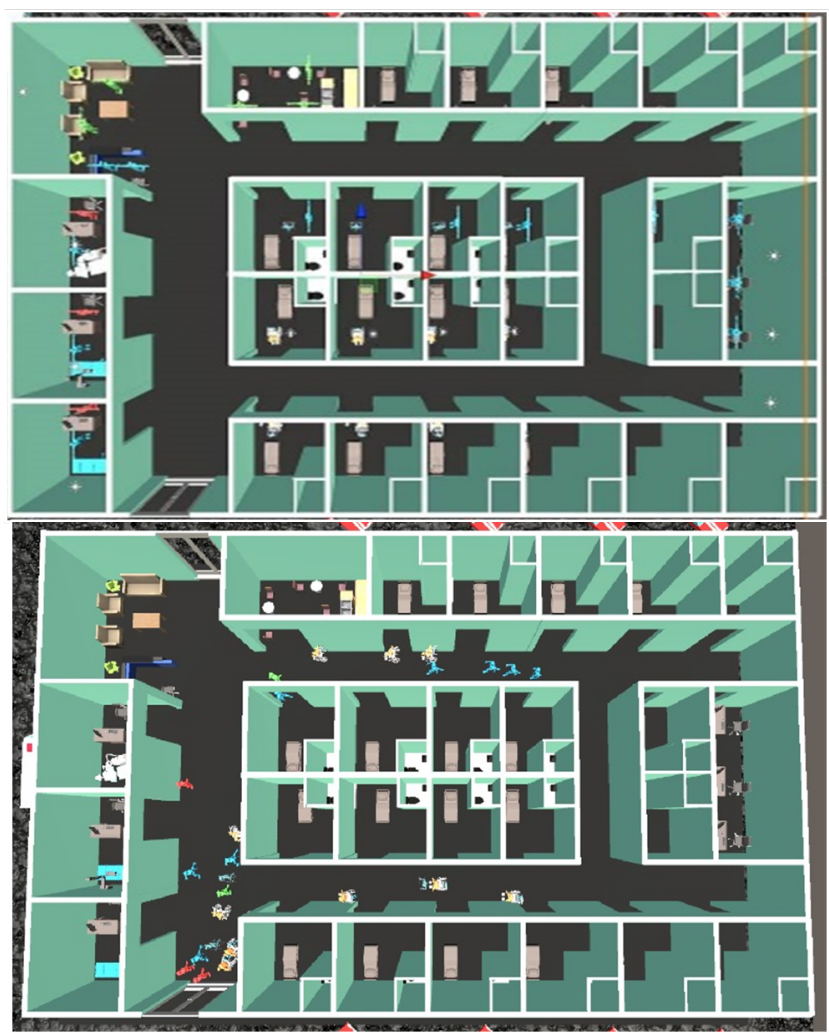

Fig. 5. The Initial Position of Agents (Top), The Final Position (Bottom) of the Agents During Simulation.

impacted their evacuation time. From Table III it can be seen the standard deviation of the evacuation time of each agent is higher than experiment 1 . This result provides an important finding. While simulating without using the FLC the exit is selected solely based on the distance. So, the agents always select the nearest exit without considering other factors which is not the case in real life evacuations. From the reviewed literature it can be seen that humans consider many factors (e.g., the exit being in the range of vision, availability of light in the surrounding, familiarity with the structure, exit familiarity) while choosing an exit for evacuation [1], [17]-[22]. This issue 
is solved when the FLC is used as the FLC considers not only the distance but also the visibility and the familiarity of the exits. Our proposed method is able to reflect and demonstrate human uncertainty and randomness to some extent. Hence, this research demonstrates that considering only distance is just not enough to select the exit. Including other factors (e.g., visibility, familiarity) help to make the evacuation simulation more realistic and increase the evacuation performance.

\section{CONCLUSION}

The objective of this research is to model and simulate assisted hospital [15] evacuation by modelling exit selection for individual agents (doctors, nurses, patients, or visitors) using FLC. For this research, only three types of transport devices have been simulated. Our research findings demonstrate that the proposed FLC can reflect human randomness and uncertainty to some extent.

However, the research has some limitations. For example, the exit is selected only once for each agent. This approach is not very feasible as the visibility of the exits are constantly changing in a practical situation. A more dynamic solution is needed for the selection procedure to properly reflect the change in visibility and the effect it causes in exit selection. Another limitation of the proposed FLC is that it only reflects the effect of three factors. However, in reality humans consider many other factors while selecting the proper exit which are not included in the proposed FLC.

Other movement devices should be considered during simulation to imitate real life situations such as stretchers and rescue sheets. Moreover, multiple floors and intermediate exits should be added to the simulation to analyze its effect in evacuation performance. Finally, the effect of social distancing should also be considered in the simulation model as social distancing is a must in the time of pandemic.

\section{ACKNOWLEDGMENT}

This research is supported by the FRGS 2019 Grant: FRGS/1/2019/ICT02/UIAM/02/2 awarded by the Ministry of Education Malaysia. The first author would like to thank IIUMTFW-2020 Scheme for sponsoring his postgraduate study

\section{REFERENCES}

[1] S. M. Lo, H.-C. Huang, P. Wang, and K. K. Yuen, "A game theory based exit selection model for evacuation," Fire Safety Journal, vol. 41, no. 5, pp. 364-369, 2006.

[2] S. M. Lo and Z. Fang, "A spatial-grid evacuation model for buildings," Journal of Fire Sciences, vol. 18, no. 5, pp. 376-394, 2000.

[3] G. S. Zhi, S. M. Lo, and Z. Fang, "A graph-based algorithm for extracting units and loops from architectural floor plans for a building evacuation model," Computer-Aided Design, vol. 35, no. 1, pp. 1-14, 2003.

[4] S. M. Lo, Z. Fang, P. Lin, and G. S. Zhi, "An evacuation model: the sgem package," Fire Safety Journal, vol. 39, no. 3, pp. 169-190, 2004.

[5] P. A. Thompson and E. W. Marchant, "A computer model for the evacuation of large building populations," Fire safety journal, vol. 24, no. 2, pp. 131-148, 1995.

[6] _ "Computer and fluid modelling of evacuation," Safety Science, vol. 18, no. 4, pp. 277-289, 1995.

[7] M. Owen, E. R. Galea, and P. Lawrence, "Advanced occupant behavioural features of the building-exodus evacuation model," Fire Safety Science, vol. 5, pp. 795-806, 1997.
[8] N. Ketchell, S. Cole, D. M. Webber, C. A. Marriott, P. J. Stephens, I. R. Brearley, J. Fraser, J. Doheny, and J. Smart, The EGRESS code for human movement and behaviour in emergency evacuations. University of Edinburgh, Artificial Intelligence Applications Institute, 1993.

[9] N. Ketchell, G. J. Bamford, and B. Kandola, "Evacuation modelling: a new approach," 1995, pp. 499-505.

[10] T. Korhonen, S. Hostikka, S. Heli, and H. Ehtamo, "Fds+evac: An agent based fire evacuation model," 0 .

[11] A. Hunt, "Simulating hospital evacuation," no. January, p. 326, 2016.

[12] V. Alonso-Gutierrez and E. Ronchi, "The simulation of assisted evacuation in hospitals," Femtc 2018, 2018.

[13] D. Ursetta, A. D'Orazio, L. Grossi, G. Carbotti, S. Casentini, and L. Poggi, "Egress from a hospital ward: A case study," Fire and Evacuation Modelling Technical Conference, 2014.

[14] A. Rahouti, R. Lovreglio, C. Dias, and S. Datoussaïd, "Simulating assisted evacuation using unity3d," 2019, pp. 265-275.

[15] S. Cao, L. Fu, and W. Song, "Exit selection and pedestrian movement in a room with two exits under fire emergency," Applied Mathematics and Computation, vol. 332, pp. 136-147, 2018.

[16] J. Gao, J. He, and J. Gong, "A simplified method to provide evacuation guidance in a multi-exit building under emergency," Physica A: Statistical Mechanics and its Applications, vol. 545, p. 123554, 2020.

[17] S. Liu, L. Yang, T. Fang, and J. Li, "Evacuation from a classroom considering the occupant density around exits," Physica A: Statistical Mechanics and its Applications, vol. 388, no. 9, pp. 1921-1928, 2009.

[18] M. Kinateder, B. Comunale, and W. H. Warren, "Exit choice in an emergency evacuation scenario is influenced by exit familiarity and neighbor behavior," Safety science, vol. 106, pp. 170-175, 2018.

[19] R. Alizadeh, "A dynamic cellular automaton model for evacuation process with obstacles," Safety Science, vol. 49, no. 2, pp. 315-323, 2011.

[20] D. C. Duives and H. S. Mahmassani, "Exit choice decisions during pedestrian evacuations of buildings," Transportation Research Record, vol. 2316, no. 1, pp. 84-94, 2012.

[21] G. Antonini, M. Bierlaire, and M. Weber, "Discrete choice models of pedestrian walking behavior," Transportation Research Part B: Methodological, vol. 40, no. 8, pp. 667-687, 2006.

[22] E.-W. Augustijn-Beckers, J. Flacke, and B. Retsios, "Investigating the effect of different pre-evacuation behavior and exit choice strategies using agent-based modeling," Procedia Engineering, vol. 3, pp. 23-35, 2010.

[23] S. Cao, L. Fu, P. Wang, G. Zeng, and W. Song, "Experimental and modeling study on evacuation under good and limited visibility in a supermarket," Fire Safety Journal, vol. 102, pp. 27-36, 122018.

[24] R. Y. Guo and H. J. Huang, "Logit-based exit choice model of evacuation in rooms with internal obstacles and multiple exits," Chinese Physics B, vol. 19, no. 3, p. 030501, 32010.

[25] R. Lovreglio, A. Fonzone, L. Dell'Olio, and D. Borri, "A study of herding behaviour in exit choice during emergencies based on random utility theory," Safety Science, vol. 82, pp. 421-431, 2016.

[26] J. Wang, L. Zhang, Q. Shi, P. Yang, and X. Hu, "Modeling and simulating for congestion pedestrian evacuation with panic," Physica A: Statistical Mechanics and its Applications, vol. 428, pp. 396-409, 2015.

[27] Y. Xu, H.-j. Huang, and L.-j. Tian, "Simulation of exit choosing in pedestrian evacuation using a cellular automaton model based on surrounding pedestrian density," 2011, pp. 1109-1112.

[28] H.-J. Huang and R.-Y. Guo, "Static floor field and exit choice for pedestrian evacuation in rooms with internal obstacles and multiple exits," Physical Review E, vol. 78, no. 2, p. 21131, 2008.

[29] S. Gwynne, E. R. Galea, P. J. Lawrence, M. Owen, and L. Filippidis, "Adaptive decision-making in response to crowd formations in building exodus," Journal of Applied Fire Science, vol. 8, no. 4, pp. 301-325, 1999.

[30] V. Schneider, "Modelling of human response and behaviour in complex surroundings," 2004

[31] H. Zhao and Z. Gao, "Reserve capacity and exit choosing in pedestrian evacuation dynamics," Journal of Physics A: Mathematical and Theoretical, vol. 43, no. 10, p. 105001, 2010. 
(IJACSA) International Journal of Advanced Computer Science and Applications,

TABLE I. RULE-BASE OF THE PROPOSED FLC

\begin{tabular}{|c|c|c|c|c|c|c|c|c|c|c|c|c|}
\hline Familiarity & \multicolumn{4}{|c|}{ NF } & \multicolumn{4}{|c|}{ F } & \multicolumn{4}{c|}{ VF } \\
\hline Distance & \multirow{2}{*}{ Visibility } & ND & FD & VFD & VND & ND & FD & VFD & VND & ND & FD & VFD \\
\hline LV & H & MH & L & ML & VH & H & MH & L & O & VH & H & MH \\
\hline MV & MH & L & ML & VL & H & MH & L & ML & VH & H & MH & L \\
\hline HV & L & ML & VL & Z & MH & L & ML & VL & H & MH & L & ML \\
\hline
\end{tabular}

TABle II. Evacuation Time of the Selected Agents (In Seconds) without using the Proposed FlC

\begin{tabular}{|c|c|c|c|c|c|c|c|c|c|c|}
\hline & \multicolumn{10}{|c|}{ Agent Name } \\
\hline & doctor 2 & nurse 11 & nurse 13 & patient with crutches 1 & patient with crutches 5 & patient with nurse 8 & patient with nurse 9 & patient with wheelchair 2 & patient with wheelchair 5 & visitor 3 \\
\hline Iteration 1 & 9.800118 & 5.635603 & 10.97213 & 9.990309 & 24.4834 & 10.97213 & 15.17973 & 17.25896 & 28.21373 & 11.28892 \\
\hline Iteration 2 & 9.716181 & 5.587833 & 10.84643 & 9.989389 & 24.49657 & 10.97705 & 15.10169 & 16.99366 & 28.18499 & 10.97705 \\
\hline Iteration 3 & 9.770281 & 5.758615 & 10.89974 & 10.05894 & 24.57523 & 11.02906 & 15.22316 & 17.1451 & 28.33407 & 11.1489 \\
\hline Iteration 4 & 9.711522 & 5.607227 & 10.89141 & 10.14683 & 24.72237 & 11.03564 & 15.1421 & 17.17188 & 28.43073 & 11.19462 \\
\hline Iteration 5 & 9.894517 & 5.7282 & 10.82855 & 9.894517 & 24.79922 & 11.01106 & 15.20018 & 17.237 & 28.28679 & 11.49648 \\
\hline Iteration 6 & 9.603971 & 5.838264 & 10.90553 & 9.714827 & 24.3032 & 11.01818 & 15.00109 & 16.95982 & 28.04262 & 10.90553 \\
\hline Iteration 7 & 9.65391 & 5.683241 & 10.84098 & 9.987705 & 24.38693 & 10.96639 & 15.11619 & 17.12051 & 28.19271 & 11.31287 \\
\hline Iteration 8 & 9.61427 & 5.75701 & 10.80946 & 9.976979 & 24.27111 & 10.95858 & 15.07268 & 16.99114 & 28.01997 & 10.95858 \\
\hline Iteration 9 & 9.618695 & 5.666526 & 10.93444 & 10.05021 & 24.62155 & 10.93444 & 15.21281 & 17.07364 & 28.23475 & 11.1718 \\
\hline Iteration 10 & 9.673256 & 5.837012 & 10.91276 & 9.827637 & 24.40374 & 11.05625 & 15.12542 & 17.03004 & 28.07324 & 11.05625 \\
\hline Standard Deviation & 0.088822 & 0.08433 & 0.048783 & 0.117239 & 0.165068 & 0.037428 & 0.065905 & 0.100012 & 0.123954 & 0.174194 \\
\hline
\end{tabular}

TABle iII. Evacuation Time of the Selected Agents (In Seconds) using the Proposed FLC

\begin{tabular}{|c|c|c|c|c|c|c|c|c|c|c|}
\hline & \multicolumn{10}{|c|}{ Agent Name } \\
\hline & doctor 2 & nurse 11 & nurse 13 & patient with crutches 1 & patient with crutches 5 & patient with nurse 8 & patient with nurse 9 & patient with wheelchair 2 & patient with wheelchair 5 & visitor 3 \\
\hline Iteration 1 & 9.599457 & 5.165013 & 10.43153 & 16.64089 & 23.92897 & 19.12175 & 23.48237 & 16.52794 & 27.73574 & 10.57593 \\
\hline Iteration 2 & 14.29306 & 5.245591 & 15.24546 & 9.359359 & 23.90145 & 10.49932 & 14.70391 & 24.97406 & 27.9697 & 11.22695 \\
\hline Iteration 3 & 13.67541 & 5.218663 & 10.47915 & 16.58311 & 29.334 & 19.2174 & 14.64088 & 16.58311 & 27.39561 & 10.2729 \\
\hline Iteration 4 & 9.539491 & 5.217213 & 10.69946 & 16.53998 & 23.81956 & 10.49263 & 14.64291 & 16.53998 & 27.79864 & 10.36195 \\
\hline Iteration 5 & 9.663138 & 5.343233 & 14.33399 & 9.289529 & 29.65339 & 10.46342 & 23.68378 & 16.85125 & 34.72333 & 13.04313 \\
\hline Iteration 6 & 9.554058 & 5.295016 & 10.10942 & 9.554058 & 24.06516 & 10.382 & 23.66674 & 16.69532 & 34.06012 & 11.33598 \\
\hline Iteration 7 & 9.626184 & 5.279238 & 10.14894 & 9.505378 & 24.01919 & 10.49212 & 14.84437 & 16.70228 & 27.86506 & 10.73832 \\
\hline Iteration 8 & 9.560124 & 5.197507 & 10.42999 & 9.716187 & 24.26485 & 19.21355 & 23.93615 & 16.82404 & 34.94345 & 12.48743 \\
\hline Iteration 9 & 9.534514 & 14.91878 & 15.64993 & 9.717838 & 24.51029 & 10.43473 & 14.91878 & 16.78667 & 34.48695 & 11.68569 \\
\hline Iteration 10 & 14.40427 & 5.121847 & 15.47817 & 9.444882 & 29.55148 & 19.27566 & 14.97395 & 25.05231 & 34.71779 & 14.81874 \\
\hline $\begin{array}{l}\text { Standard } \\
\text { Deviation }\end{array}$ & 2.089058 & 2.906826 & 2.37543 & 3.2450102 & 2.500984 & 4.2851 & 4.364833 & 3.331497 & 3.426042 & 1.360217 \\
\hline
\end{tabular}

[32] W. Yuan and K. H. Tan, "An evacuation model using cellular automata," Physica A: Statistical Mechanics and its Applications, vol. 384, no. 2, pp. 549-566, 2007.

[33] T. Huan-Huan, D. Li-Yun, and X. Yu, "Influence of the exits' configuration on evacuation process in a room without obstacle," Physica A: Statistical Mechanics and its Applications, vol. 420, pp. 164-178, 2015.

[34] D. Li-Yun, C. Li, and D. Xiao-Yin, "Modeling and simulation of pedestrian evacuation from a single-exit classroom based on experimental features," Acta Physica Sinica, vol. 64, no. 22, 2015.

[35] B. L. Mesmer and C. L. Bloebaum, "Incorporation of decision, game, and bayesian game theory in an emergency evacuation exit decision model," Fire Safety Journal, vol. 67, pp. 121-134, 2014.

[36] H. A. Kurdi, S. Al-Megren, R. Althunyan, and A. Almulifi, "Effect of exit placement on evacuation plans," European Journal of Operational Research, vol. 269, no. 2, pp. 749-759, 2018.

[37] X. Yang, X. Yang, and Q. Wang, "Pedestrian evacuation under guides in a multiple-exit room via the fuzzy logic method," Communications in Nonlinear Science and Numerical Simulation, vol. 83, p. 105138, 4 2020.

[38] T. Liu, X. Yang, Q. Wang, M. Zhou, and S. Xia, "A fuzzy-theory-based cellular automata model for pedestrian evacuation from a multiple-exit room," IEEE Access, vol. 8, pp. 106 334-106345, 2020.

[39] W.-L. Wang, F.-F. Wan, and S.-M. Lo, "Game theory model of exit selection in pedestrian evacuation considering visual range and choice firmness*," Chinese Physics B, vol. 29, no. 8, p. 084502, 72020.

[40] D. Zhang, G. Huang, C. Ji, H. Liu, and Y. Tang, "Pedestrian evacuation modeling and simulation in multi-exit scenarios," Physica A: Statistical Mechanics and its Applications, vol. 582, p. 126272, 112021.

[41] Y. Fu, W. Shi, Y. Zeng, H. Zhang, X. Liu, and Y. Liu, "Simulation study on pedestrian evacuation optimization in a multi-exit building," vol. 1780, no. 1, 2021, p. 12024.

[42] D. Xu, X. Huang, J. Mango, X. Li, and Z. Li, "Simulating multi-exit evacuation using deep reinforcement learning," Transactions in GIS, vol. 25, no. 3, pp. 1542-1564, 62021.

[43] A. Edrisi, B. Lahoorpoor, and R. Lovreglio, "Simulating metro station evacuation using three agent-based exit choice models," Case Studies on Transport Policy, vol. 9, no. 3, pp. 1261-1272, 92021.

[44] G. Ma, Y. Wang, and S. Jiang, "Optimization of building exit layout: Combining exit decisions of evacuees," Advances in Civil Engineering, vol. 2021, 2021.

[45] L. Fu, J. Fang, S. Cao, and S. Lo, "A cellular automaton model for exit selection behavior simulation during evacuation processes," Procedia Engineering, vol. 211, pp. 169-175, 12018.

[46] S. P. Hoogendoorn and P. H. L. Bovy, "Pedestrian route-choice and activity scheduling theory and models," Transportation Research Part B: Methodological, vol. 38, no. 2, pp. 169-190, 2004.

[47] G. Santos and B. E. Aguirre, "A critical review of emergency evacuation simulation models." NIST, Gaithersburg, USA, 2004, p. 339.

[48] H. L. Kluepfel, "A cellular automaton model for crowd movement and egress simulation,” Ph.D. dissertation, 2012.

[49] Z. He, M. Shi, and C. Li, "Research and application of path-finding algorithm based on unity 3d," 2016 IEEE/ACIS 15th International Conference on Computer and Information Science, ICIS 2016 - Proceedings, 82016.

[50] MAMDANI and E. H., "Applications of fuzzy algorithms for control of simple dynamic plant," Proc. IEE, vol. 121, pp. 1585-1588, 1974.

[51] L. Fu, W. Song, and S. Lo, "A fuzzy-theory-based behavioral model for studying pedestrian evacuation from a single-exit room," Physics Letters A, vol. 380, no. 34, pp. 2619-2627, 82016. 